\title{
Mouvements de seiches à trois dimensions
}

\section{Tridimensional seiche motion}

\author{
PAR F. BIESEL ET B. LE MEHAUTÉ
}

INGÉNIEURS AU LABORATOIRE DAUPhINOIS n'HYDRAULIQUe (SOGREAH, GrenobLe)

\footnotetext{
- Considération générale sur la résonance des plans d'eau plus ou moins ouverts excitée par une onde périodique venant du large. Définitions de la résonance. Corréction d'ouverture.

- Résonance des plans d'eau fermés. Position mathématique du problème du mouvement propre des plans d'eau. Cas des plans d'eau de profondeur variable et de parois limites verticales ou obliques. Création et entretien des oscillations.

- Résonance des plans d'eau ouverts localement.

- Résonance des plans d'eau largement ouverts.

- Influence de l'incidence des ondes excitatrices dans les cas d'une grande ouverture et d'une ouverture de largeur moyenne.
}

\begin{abstract}
- A general treatment of resonance in open or partially exposed water surfaces excited by periodic waves emanating from open sea. Resonance defined. Exposure correction.

- Resonance in enclosed water surfaces. The mathematical aspect of the problem of the proper motion of water surfaces. The case of water, the depth of which varies and which has vertical or oblique bounding walls. Setting up and maintaining oscillations.

- Resonance in locally exposed water surfaces.

- Resonance in greatly exposed water surfaces.

- The effect of the incidence of exciting waves for great and medium exposures.
\end{abstract}

\section{I. - GENERALITES}

Dans nos études antérieures sur le comportement de la houle sur certains obstacles [1] et sur les mouvements de seiches dans une darse rectangulaire [2], [3], nous nous sommes limités aux mouvements à deux dimensions ou pouvant, en première approximation, être considérés comme tels. Il est clair que ces études sont insuffisantes pour traiter la généralité des cas. Souvent les ports et les bassins eux-mêmes ont des dimensions transversales du mème ordre de grandeur que leurs dimensions longitudinales et rien ne permet de supposer que les mouvements d'oscillation se font suivant certaines directions favorisées. Ceci est encore plus vrai dans le cas des baies et des plateaux continentaux dont le rôle peut être important et qui doivent parfois être représentés sur les modèles réduits [4], [5].
L'exploitation de ces derniers nécessite également des connaissances générales étendues sur le comportement des plans d'eau de forme quelconque au point de vue des résonances et des oscillations possibles.

Nombre des notions acquises au cours de l'étude bi-dimensionnelle sont encore valables pour les problèmes à trois dimensions. On rencontre également des résonances dans des plans d'eau ouvrant sur le large par une ouverture ou plus généralement par une « combinaison d'obstacles 》 - le mot « obstacle 》 ayant, comme nous l'avons vu, un sens très général. Les résonances les plus accentuées se créent souvent lorsque l'ouverture possède elle-même une dimension relativement faible, les autres parois limitant le plan d'eau étant bien entendu réfléchis- 
santes. On se souvient aussi que, dans le cas d'une darse «sous ouverte», la résonance maximum se produit pour une longueur légèrement différente de la demi-longueur d'onde; de même les mouvements les plus violents ne correspondent pas toujours exactement aux fréquences propres du plan d'eau considéré comme fermé.

Il s'introduira done une notion de « correction d'ouverture ». On peut d'ailleurs noter que, du simple point de vue géométrique, la facon de "fermer » un plan d'eau se prête à un certain arbitraire. Considérons par exemple la figure 1 représentant un bassin rectangulaire ouvert en un de ses coins. Les tracés en pointillé représentent divers « contours de fermeture » également plausibles. Pour chacun des plans d'eau ainsi délimités on aura des périodes propres différentes. Par conséquent les corrections d'ouverture qu'il y aura lieu d'appliquer seront différentes suivant que l'on se référera à un mode de fermeture plutôt qu'à un autre. En vue de ce qui précède, il est peut-être raisonnable de chercher à exprimer la correction d'ouverture par un tracé

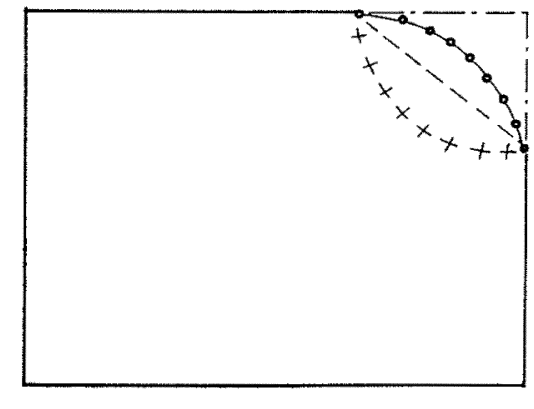

FIG. 1

La façon de fermer un plan d'eau pour en déterminer les périodes propres se prête à un certain arbitraire.

de fermeture tel que celui marqué par des croix sur la figure 1. Ce dernier tracé étant choisi non pour se raccorder au mieux avec l'allure des parois du port, mais pour délimiter un plan d'eau fermé ayant la fréquence de résonance du plan d'eau ouvert.

En fait, il est à prévoir que les tracés ainsi définis différeront peu entre eux lorsque l'ouverture sera petite. Pratiquement, lorsque les dimensions du port seront grandes par rapport à celles de l'entrée (et que l'on n'étudiera pas les harmoniques d'ordre élevé), la « correction d'ouverture » pourra devenir négligeable ou, en d'autres termes, n'importe quel contour de fermeture raisonnable (un segment de droite par exemple) pourra être pris en considération.

Rappelons que nous avons défini les mouvements 《 résonnants» comme étant ceux correspondant aux maxima de la courbe qui donne l'agitation en fonction de la période d'une onde excitatrice d'amplitude constante. Ainsi que nous venons de le rappeler, on obtient ces maxima pour des périodes légèrement plus faibles que celles correspondant aux périodes propres du plan d'eau considéré comme complètement fermé à son entrée.

Il importe de préciser que notre définition diffère de celle adoptée par le professeur McNown qui identifie les périodes de résonance avec celles des mouvements propres des plans d'eau considérés comme fermés [6]. Suivant cette définition, nous pourrons rencontrer des mouvements non résonnants » pour lesquels l'agitation dans la darse sera supérieure à celle des mouvements résonnants de périodes voisines.

La figure 2, extraite de la thèse du professeur McNown, représente des répartitions d'amplitude le long du diamètre passant par l'entrée d'un port circulaire. Celles qui sont marquées

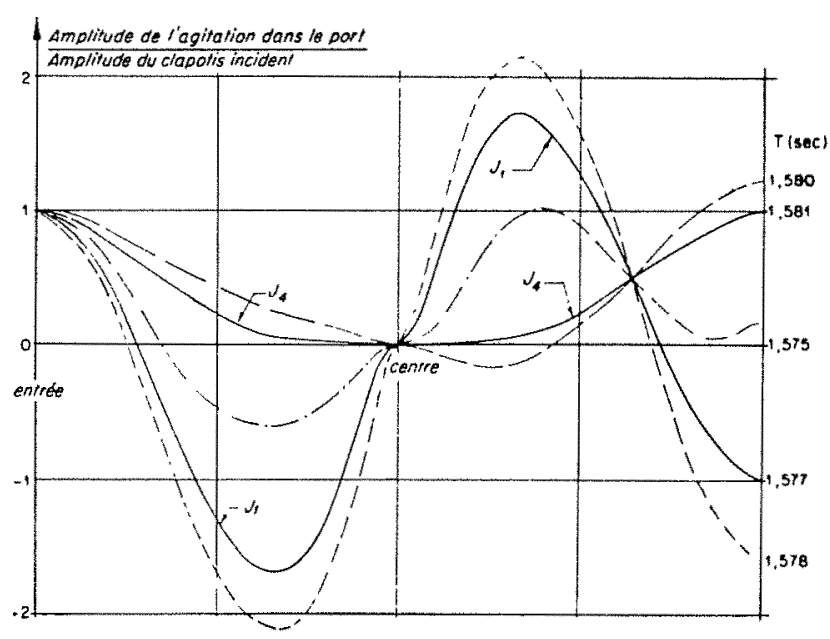

FIG. 2

Répartition des amplitudes dans un port circulaire : les mouvements « non résonnants 》 peuvent avoir une amplitude supérieure à l'amplitude des mouvements «résonnants» $J_{1}$ et $J_{t}$ (d'après MeNown).

$J_{1}$ et $J_{4}$ sont des mouvements de résonance, les autres sont des mouvements non résonnants. On peut constater sur celte figure que les mouvements résonnants au sens du professeur McNown ne correspondent pas au maximum d'agitation.

Ceci peut être interprété comme étant la conséquence d'un choix arbitraire, c'est-à-dire ne tenant pas compte de la correction d'ouverture, des limites fermant l'entrée du port. Ceci nous confirme que la définition même des conditions de résonance pose un problème délicat, ainsi que nous l'avions mentionné plus haut.

La définition donnée par le professeur McNown a l'avantage d'être très précise, à condition toutefois que l'on ait arrêté un mode de fermeture du plan d'eau. La définition que nous 
avons utilisée est peut-être plus conforme aux exigences de la pratique puisqu'elle se réfère au maximum d'agitation, il se peut toutefois que dans certains cas elle souffre d'ambiguïté pour les mouvements à trois dimensions. En effet, pour ce cas, non seulement les amplitudes mais aussi les configurations changent lorsque la période varie; ainsi il peut être difficile de comparer les amplitudes de deux mouvements d'allure très différente.

De plus, il faut remarquer (et ceci est extrêmement important pour la pratique) qu'il peut y avoir en certains points des agitations particulièrement accentuées, sans que pour cela on puisse à proprement parler de résonance. Ces renforcements peuvent être dus à des concentrations résultant par exemple de variations de profondeur (réfraction) ou de la disposition de parois formant convergent. Par exemple, lorsque le professeur McNown observe que le mouvement radial de l'eau dans un port circulaire de profondeur constante a, au centre, une amplitude égale à 1,76 fois l'amplitude du clapotis total extérieur, cela n'est-il pas dû à la convergence radiale des trajectoires des particules d'eau, plus qu'à une "résonance» au sens habituel du mot?

Pour clore cette comparaison entre les diverses définitions de la résonance, rappelons que celle donnée par McNown équivaut à caractériser les mouvements résonnants par la nullité des vitesses normales aux limites définissant la passe d'entrée.

Nous avons vu au contraire, dans le cas de ce que nous avons appelé mouvements résounants bi-dimensionnels, que les vitesses dans la passe d'entrée étaient toujours très élevées. N'ous avons aussi constaté ce même fait sur des mouvements résonnants à trois dimensions.

Ce préambule nous montre que l'utilisation des notions acquises dans les études précédentes [2], [3] sera dangereuse tant que l'on n'aura pas reconnu avec un certain détail quelles sont les différences essentielles de comportement entre les mouvements à trois dimensions et les mouvements à deux dimensions. C'est dans cet esprit qu'est écrit cet article qui, loin de viser à une étude générale et complète des mouvements à trois dimensions, a pour but de poser quelques jalons qui, joints à l'intuition et au sens des phénomènes hydrauliques, pourront aider les chercheurs à se faire une représentation utile des phénomènes.

Ainsi que cela a déjà été exposé [2], [3], l'étude des mouvements de résonance accentuée, qui en pratique sont les plus intéressants, se ramène en général à celle des mouvements propres des plans d'eau. Nous avons vu, par exemple, que les darses allongées entraient en résonance accentuée quand elles étaient «très fermées » et que cette résonance était alors très voisine de la résonance «demi-onde » correspondant au cas d'une darse complètement fermée. Nous avons vu également que dans le cas des darses «sur-ouvertes » la résonance maximum se produisait au voisinage du «quart d'onde ». On retrouve des résultats semblables pour les mouvements à trois dimensions et de même il convient de distinguer entre les plans d'eau presque entièrement fermés et ceux largement ouverts. Nous concentrerons notre attention sur le cas des plans d'eau très fermés et en particulier sur le cas limite des plans d'eau entièrement fermés qui est celui par exemple des lacs. Pour ce cas nous serons ramenés, ainsi que nous l'avons indiqué par ailleurs [7], à établir une analogie avec l'étude des oscilla. tions propres d'un milieu à deux dimensions, la troisième dimension, en l'occurrence la profondeur, pouvant être en général éliminée des équations. Après cette étude nous examinerons l'influence que peuvent avoir les formes et les dispositions des ouvertures mettant le plan d'eau en contact avec le milieu exlérieur sur l'excitation, l'amplitude et la période des mouvements de résonance. Enfin, nous étudierons l'influence possible de Ia direction d'incidence des ondes de seiches excitatrices.

\section{II. - RESONANCES DE PLANS D'EAU FERMÉS}

\section{1) Profondeur constante :}

Le problème des oscillations d'une masse d'eau de profondeur constante $h$ dans une enceinte fermée à parois verticales a été depuis longtemps étudié et mis en équations [8], [9]. Le potentiel du mouvement de l'eau est donné par la formule :

$$
\varphi=\varphi_{1} \operatorname{ch} k(z+h) e^{i \sigma t}
$$

$\varphi_{1}$ est une fonction de $x$ et $y$, telle que :

$$
\frac{\partial^{2} \varphi_{1}}{\partial x^{2}}+\frac{\partial^{2} \varphi_{1}}{\partial y^{2}}+k^{2} \varphi_{1}=0
$$

les formes de $\varphi_{1}$ et les valeurs admissibles de $k$ sont déterminées par l'équation ci-dessus et par la condition :

$$
\frac{\partial \varphi_{1}}{\partial n}=0
$$


valable sur les parois verticales entourant le plan d'eau. A chaque valeur propre $k$ correspond une fréquence donnée par la formule :

$$
\sigma^{2}=g k \text { th } k h
$$

Pour cette valeur, l'élévation est donnée par :

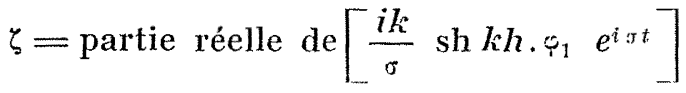

Les solutions de ce problème ne sont connues d'une facon complète que pour de rares cas particuliers. Il est en principe possible de les calculer dans le cas général par des méthodes numériques souvent très longues. En fait, cela ne présentera pas d'intérêt pratique car l'étude des cas particuliers bien connus suffit pour dégager les principes essentiels valables dans ce genre de problème.

On démontre par exemple que les fonctions propres sont données par les formules :

$$
\varphi_{1}=\mathrm{J}_{n}(\mathrm{kr}) \cos n \Theta
$$

pour les ports circulaires (coordonnées polaires),

$$
\varphi_{1}=\sin \frac{\pi m x}{a} \sin \frac{\pi n y}{b},
$$

pour les ports rectangulaires de côtés $a$ et $b$ (coordonnées rectangulaires).

Rappelons que dans le cas des oscillations longitudinales à deux dimensions d'un canál de longueur $d$, la fonction correspondante a la valeur :

$$
\varphi_{1}=\sin \frac{\pi m x}{d}
$$

On constate tout de suite que, dans les premiers cas, les fonctions propres dépendent de deux paramètres $k$ et $n, m$ et $n$, tandis que dans le cas d'un canal rectiligne, elles ne dépendent plus que d'un paramètre $m$. On peut en déduire d'une façon un peu superficielle, mais qui peut être précisée par les études mathématiques de ces cas particuliers, que le nombre des types de résonances possibles est beaucoup plus élevé pour des ports circulaires ou rectangulaires que pour des canaux allongés, la longueur de ces derniers étant de l'ordre de grandeur des dimensions transversales des premiers. Il en résulte en particulier que l'on trouve beaucoup plus facilement des périodes de résonance extrêmement voísines, quoique correspondant à des mouvements nettement différents, pour les bassins à deux dimensions que pour les canaux où les mouvements se font seulement dans le sens longitudinal. La possibilité de rencontrer deux fréquences propres très voisines est encore augmen- tée pour les bassins du fait que la répartition de ces fréquences propres sur le spectre n'est pas aussi régulière que dans le cas des canaux. Ainsi, de telles quasi-coïncidences de fréquences propres pourront se produire même pour les fréquences relativement peu élevées, qui sont en principe les moins «serrées».

Une autre différence est que pour les bassins à trois dimensions il existe parfois plusieurs et même une infinité de modes de résonances possibles pour certaines périodes propres. Ainsi dans le cas d'une excitation ayant une telle période propre, la détermination de la part respective que prennent chacun des mouvements possibles dans l'agitation totale doit se faire d'après des considérations physiques particulières.

\section{2) Profondeur varilisile - Parois limites VERTICALES :}

Si l'on suppose que les profondeurs varient suffisamment lentement, on peut admettre qu'en chaque point les mouvements de l'eau sont régis par les équations qui seraient valables pour une profondeur constante égale à la profondeur locale. Avec cette hypothèse, on est amené à remplacer l'équation différentielle indéfinie, où $k$ était une constante, par une équation s'écri vant :

$$
\frac{\partial^{2} \varphi_{1}}{\partial x^{2}}+\frac{\partial^{2} \varphi_{2}}{\partial y^{2}}+k^{2}(x, y) \varphi_{1}=0
$$

où $k$ est lié à une constante $\sigma$ (fréquence angulaire) par l'égalité :

$$
\sigma^{2}=g k \text { th } k h
$$

$h$ étant une fonction donnée de $x$ el de $y$, les conditions aux limites restant les mêmes.

Le paramètre dont il faudrait chercher les valeurs propres serait alors $\sigma$ et Jes fonctions propres $\diamond_{1}$ donneraient, à un facteur près, les valeurs de l'amplitude en chaque point. Ce facteur ne serait sans doute pas rigoureusement constant mais serait une fonction de $h$ et en conséquence varierait lentement de même que cette srandeur.

Ces considérations permettent de conclure que les bassins de profondeur lentement variable ont qualitativement un comportement tout à fait analogue à celui des bassins de profondeur constante.

\section{3) Parois limites obliques :}

Lorsque le plan d'eau, au lieu de se terminer par des parois verticales, se termine par des parois ohliques, il est un peu plus difficile d'établir les équations du phénomène car les condi- 
tions aux limites sont plus complexes à exprimer. Néanmoins, le sens physique laisse penser que le comportement ne diffère pas en son essence de celui des cas précédemment étudiés. (Etant entendu que la pente des parois n'est pas suffisamment faible cependant pour provoquer des déferlements.)

\section{4) Création et entretien des oscillations :}

Les oscillations d'un plan d'eau doivent être créées par une cause extérieure réalisant un apport d'énergie à la masse liquide et de plus, du fait de la viscosité et de la turbulence, ces oscillations décroissent et disparaissent si rien ne vient renouveler leur énergie. Il est évidemment essentiel de connaitre les lois liant la nature de l'agitation aux caractéristiques de l'excitation. Le caractère de cette relation peut être étudié théoriquement dans un très grand nombre de cas. Cette étude permet d'énoncer des conclusions très générales et surtout de comprendre l'essence même des phénomènes en jeu. Nous allons examiner plus spécialement ces conclusions dans deux cas types : $1^{\circ}$ les oscillations sont créées par des perturbations atmosphériques agissant sur tout ou partie de la surface du plan d'eau; $2^{\circ}$ les oscillations sont créées par une perturbation localisée en un point de la périphérie du plan d'eau (entrée d'un port par exemple).

Dans le premier cas, nous avons comme données l'évolution, qui peut être très compliquée, de la configuration de la perturbation atmosphérique au cours du temps. Dans le cas général, les mouvements du plan d'eau excité par de telles perturbations résulteront de la superposition d'une déformation « statique » (en général de très faible amplitude) équilibrant la valeur instantanée des perturbations ef d'un certain nombre des oscillations propres du plan d'eau. Certaines de ces oscillations propres seront particulièrement favorisées. Ce sont d'une part celles dont la forme s'adapte particulièrement à la forme de la perturbation atmosphérique (par exemple des seiches uninodales si la perturbation atmosphérique a un gradient de pression constant perpendiculairement à une ligne nodale possible), et, d'autre part, celles dont la fréquence se trouve particulièrement représentée dans les fluctuations de la perturbation atmosphérique. II y a donc deux causes principales de renforcement préférentiel d'un mode particulier d'oscillat:on, la première étant la ressemblance de la répartition de la perturbation atmosphérique à la répartition des élévations de niveau dans l'oscillation libre et la seconde étant la copériodicité au moins approximative de l'excitation et du mouvement de seiche considéré. Dans des cas particuliers où l'excitation présentera à la fois une périodicité assez marquée et une configuration pas trop différente de celle de l'oscillation correspondante, cette oscillation pourra prendre une amplitude marquée et masquer toutes les autres. Lorsque la seiche ainsi favorisée sera une des plus longues dont le plan d'eau est susceptible, il y aura en plus un phénomène d'auto-épuration dû au fait que les seiches plus courtes s'amortissent plus rapidement. II arrivera ainsi que parfois le phénomène de seiche se présentera avec une pureté frappante.

\section{III. - RESONANCE DES PLANS D'EAU OUVERTS LOCALEMENT}

L'étude des excitations localisées en un point de la périphérie est plus simple car le facteur répartition dans l'espace n'est plus à prendre en considération. Il reste seulement à considérer la répartition des actions dans le temps et la localisation de l'excitation. Laissons de côté le cas (peu intéressant pour les seiches portuaires) d'un ćbranlement local et un:que, et concentrons notre attention sur celui de l'excitation du plan d'eau à travers une brèche ou ouverture, constituée dans les parois qui le limitent, et le mettant en communication avec un océan dans lequel se propagent des ondes de gravité. Ces ondes ont en général une périodicité assez marquée et de toutes facons on peut les considérer comme résultant de la somme d'un nombre plus ou moins grand d'ondes élémentaires ayant chacune une périodicité bien définie. Par conséquent, il est possible de simplifier l'étude de cette excitation (dans l'hypothèse toujours sous-entendue où l'on peut admettre que les phénomènes considérés sont linéaires, donc susceptibles d'addition) en ne considérant que l'excitation due à une onde individuelle de période parfaitement définie. Eventuellement il pourra être nécessaire de reconstituer l'agitation réelle par une sommation à partir de toutes les agitations élémentaires correspondant aux fréquences individuelles des trains d'ondes incidents.

Comme nous l'avons précédemment indiqué, cette étude a été faite à la fois théoriquement et expérimentalement pour les cas particuliers des plans d'eau circulaires et rectangulaires par le professeur McNown, puis par le professeur 
Kravtchenko et le docteur Apté [10] et [11]. Nous renvoyons aux thèses et articles présentés par ces auteurs, pour plus de précisions. Cependant, nous désirons souligner une des conséquences importantes en ce qui concerne la facon dont les mouvements sont excités à travers ùne ouverture.

Revenons à la figure 2, extraite de la thèse du professeur McNown. Nous avons déjà remarqué que les mouvements «non résonnants»te] que les définit l'auteur peuvent prendre une amplitude plus grande que les mouvements propres eux-mêmes. Ceci est bien en concordance avec ce que nous avons vu dans le cas des mouvements de seiches à une dimension.

Néanmoins, la nature des phénomènes est plus compliquée et l'on constatera par exemple que, lorsque la période passe de 1,577 à 1,578, on a une augmentation d'amplitude correspondant par conséquent à une augmentation de période, tandis que lorsque cette dernière passe de 1,581 à 1,580 , on a également une augmentation d'amplitude, quoique cela corresponde à une diminution de période. Nous rappelons que dans le cas des darses allongées sous-ouvertes, ce qui correspond à peu près comme mode d'excitation à celui du port circulaire étudié, les diminutions de période amenaient une augmentation de l'amplitude à l'intérieur de la darse par rapport à l'amplitude de résonance et réciproquement les augmentations de périodes amenaient une diminution. Cette règle ne peut donc pas se transposer dans le cas des mouvements à trois dimensions.

En fait, les modifications d'amplitudes par rapport aux mouvements de résonance sont dues, dans l'un et l'autre cas, à des causes très différentes. En particulier les hypothèses utilisées pour l'étude du port circulaire impliquent que les mouvements à l'intérieur et à l'extérieur du port se raccordent parfaitement de part et d'autre de l'entrée. On sait que cette hypothèse est abandonnée pour l'étude des darses allongées, puisque au cours de cette étude nous avons trouvé des cas où l'amplitude dans la darse devenait infiniment grande et par conséquent ne pouvait se raccorder avec l'amplitude extérieure.

De plus, la position de l'ouverture par rapport à la configuration du mouvement dans la darse a une importance capitale sur l'amplitude de l'agitation. C'est ainsi qu'une darse rectangulaire communiquant avec le large par une ouverture très étroite pourra avoir une agitation extrêmement grande ou nulle suivant que la passe d'entrée se situe sur une ligne ventrale ou sur une ligne nodale du mouvement résonnant dans la darse.

Enfin, notons que certains mouvements de résonance sont susceptibles d'addition lorsque les périodes de ces mouvements sont peu différentes. Considérons, en effet, le cas d'une darse rectangulaire excitée par l'un de ses coins (cas de la fig. 1). Pour une certaine période $\mathrm{T}_{1}$, on obtiendra un mouvement résonnant fondamental, parallèle à deux parois. Pour une période $\mathrm{T}_{2}=\mathrm{T}_{1}+\Delta \mathrm{T}$, on obtiendra un mouvement résonnant fondamental de même type dans un sens perpendiculaire à ce premier mouvement. Pour une période intermédiaire $\mathrm{T}_{1}-\Delta \mathrm{T} / 2$, le mouvement, sans être au maximum de résonance, se fait suivant la diagonale passant par l'entrée de la darse. Lorsque l'on tend à donner aux côtés du rectangle des valeurs presque égales, les deux types de mouvements fondamentaux restent encore très nets, mais le mouvement de période $\mathrm{T}_{1}+\Delta^{r} \mathrm{~T} / 2$ augmente d'amplitude.

Enfin, lorsque la darse est un carré parfait $\left(\mathrm{T}_{1}=\mathrm{T}_{2}\right)$, le móuvement résonnant résultant se fait suivant la diagonale passant par l'entrée.

\section{IV. - RESONANCE DES PLANS D'EAU LARGEMENT OUVERTS}

Parmi les mouvements des plans d'eau largement ouverts sur le large, il existe deux types de mouvements résonnants de caractéristiques bien différentes.

Le premier type de mouvements peut ètre rapproché du type quart d'onde. Par analogie à ce que nous avons vu pour les mouvements plan, ce type de mouvement se présente comme une oscillation en masse entre le bassin résonnant ef l'étendue du large. Il se manifeste violemment d'un côté par la concentration de l'énergie et, d'une façon insensible, du côté large par suite de l'étalement des ondes. De plus, la variation de profondeur et l'obliquité des parois provoquent parfois des amplitudes verticales extrêmement grandes.

Le deuxième type de résonance des plans d'eau largement ouvert sera du type demi-onde. Nous avons observé ce phénomène dans une darse rectangulaire ouverte sur un còté entier et excitée par une onde cheminant parallèlement à ce côté. La résonance a lieu lorsque la largeur de la 
darse (prise dans le sens de cheminement de l'onde) est égale à une demi-longueur d'onde.

Notons enfin la tendance préférentielle du mouvement à se faire suivant la direction de l'onde incidente, se traduisant souvent par une dissymétrie dans la répartition des amplitudes de mouvement apparemment plan. Ce serait, par exemple, le cas d'une darse «sur-ouverte » résonnant en quart d'onde, excitée par une onde arrivant obliquement : l'amplitude au fond en $\mathrm{A}$ serait supérieure à l'amplitude en $\mathrm{B}$ (fig. 3).

Ceci nous conduit à parler de l'influence de l'incidence des ondes excitatrices.

\section{FIG. 3}

L'onde incidente provoque dans la darse une résonance du type $1 / 4$ d'onde, avec un ventre de mouvement en $A B$. Mais l'amplitude en A est supérieure à l'amplitude en B par suite de l'obliquité de l'incidence.

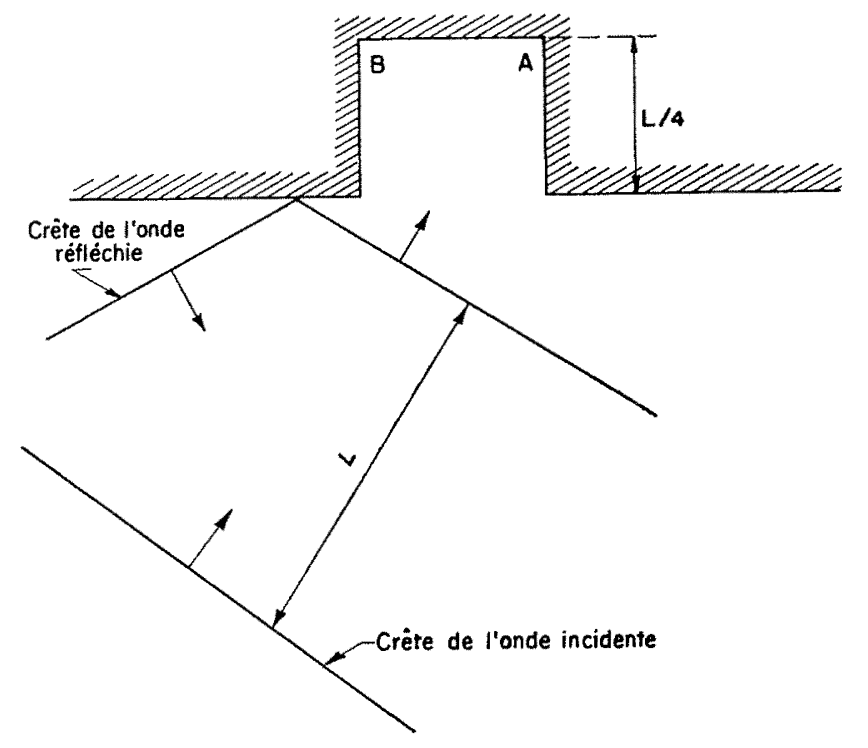

\section{V. - INFLUENCE DE L'INCIDENCE DES ONDES EXCITATRICES}

A notre connaissance, il n'existe pas d'étude théorique ou expérimentale approfondie de l'influence de l'incidence des ondes excitatrices. Cependant, ceci est évidemment important, ne serait-ce que pour la conceplion des modèles réduits. En effet, s'il était possible de se dispenser de reproduire sur ces modèles les différentes directions d'où les ondes peurent venir et de mener toutes les études avec une seule direction, ceci conduirait à une économie très importante tant sur la construction du modèle que sur son exploitation. Il est donc essentiel de savoir jusqu'à quel point la direction des ondes incidentes influe sur la nature de l'agitation que l'on veut étudier. Dans l'état actuel de nos connaissances, et à l'aide de quelques essais effectués sur ce. sujet au Laboratoire Dauphinois d'Hydraulique, il est possible de tirer des conclusions pour un certain nombre de cas particuliers. C'est ce que nous allons examiner ci-dessous :

\section{1) OUVertTtre très ÉtroIte :}

Dans ce cas, le niveau de l'eau à l'extérieur du port ne varie sensiblement pas de l'un à l'autre des musoirs définissant l'ouverture. Les échanges à travers cette ouverture dépendent essentiellement des différences de niveau de part et d'autre de celle-ci et enfin, ces échanges s'effectuant sur une largeur très faible, ne réagissent pratiquement pas sur l'état du milieu extérieur qui se comporte à peu près comme si l'ouverture était inexistante. On peut dire que l'action de la houle incidente dépend uniquement de l'amplitude qu'elle crée à l'entrée du plan d'eau étudié et non directement de son incidence. Cependant, l'amplitude à l'entrée du plan d'eau dépend souvent de l'incidence des ondes excitatrices par suite des conditions topographiques existant au voisinage de l'entrée. Prenons un exemple simple pour bien faire comprendre ce point de vue: supposons que l'ouverture soit pratiquée dans un quai vertical parfaitement réfléchissant, rectiligne, de longueur infinie et bordant un océan également indéfini. Si la direction de propagation des houles est parallele à ce mur, l'amplitude créée au voisinage de l'entrée est égale à celle de la houle. Par contre, si les houles viennent d'autres directions, par suite du phénomène de réflexion sur le mur, l'amplitude créée au voisinage de l'entrée est le double de celle de la houle incidente. Ainsi, dans ce cas, on voit que l'incidence a eu un effet direct sur la nature de l'excitation pénétrant par l'ouverture.

Notons que la discontinuité qui existe dans l'exemple précédent entre des houles parallèles au mur et des houles faisant avec celui-ci un angle aussi petit que l'on veut, disparaît dans les cas réels où le mur n'est pas infiniment long et où les phénomènes de diffraction interviennent pour rétablir la continuité.

En conclusion, dans l'étude des oscillations de plans d'eau s'ouvrant sur la mer par une ouverture très étroite, l'incidence des houles excitatrices doit être prise en compte dans la mesure où elle réagit sur la valeur de l'amplitude créée au droit de l'ouverture. Cette nécessité amène à considérer l'évolution de la houle au voisinage des côtes et par conséquent le détail des formes 
de celles-ci et de leurs propriétés vis-à-vis de la réflexion ou de la réfraction des ondes incidentes.

Une autre conséquence des considérations précédentes, dans le cas où la côte présente des baies ou des anfractuosités de formes diverses, c'est qu'il faut considérer l'influence de l'incidence sur la résonance dans ces baies ou anfractuosités; ccci nous ramène par conséquent à l'étude des plans d'eau largement ouverts.

\section{2) Grande ouverture :}

Pour le cas des ouvertures très larges, il est essentiel de tenir compte de l'incidence des ondes excitatrices. On peut parfois traiter le problème en appliquant les méthodes connues sous le nom de « diagramme de réfraction-réflexion». Cette méthode permet de suivre le cheminement des ondes et de ses réflexions successives sur les côtes ou autres obstacles limitant le plan d'eau. On peut ainsi étudier la manière dont l'énergie est susceptible de se concentrer en certains points.

Ceci nécessite évidemment de pouvoir estimer convenablement le pouvoir réfléchissant des côtes, ce qui n'est pas toujours commode, surtout lorsque celles-ci sont découpées. D'autre part, lorsque de nombreuses réflexions successives sont susceptibles de se créer, il devient pratiquement impossible de poursuivre les épures et l'on retrouve en fait des phénomènes de la famille de la résonance. En ce qui concerne le cas des baies largement ouvertes, nous renvoyons le lecteur au chapitre relatif au problème de similitude des études sur modèle réduit, chapitre dans lequel sont donnés plusieurs exemples d'études de concentration d'énergie due aux formes des côtes au voisinage des ports étudiés [4], [5],

\section{3) OUverture de LARgeUr MOYenNe :}

Entre les ouvertures très étroites et les ouvertures très larges qui peuvent relativement être traitées sans trop de difficultés, se trouvent les ouvertures moyennes qui, comme on peut s'y attendre, seront particulièrement délicates à étudier.

S'il est, à la rigueur, admissible de parler de très grand et de très petit sans fixer d'ordre de grandeur, cela devient tout à fait inacceptable si l'on parle de «moyen ». Il importe donc de préciser ce que l'on entend par ouverture de largeur moyenne. Nous avons vu que les ouvertures très étroites étaient caractérisées par le fait que l'amplitude de la dénivelée ne variait que très peu le long de l'ouverture. Ceci signifie que la largeur de l'ouverture doit être faible par rapport à la longueur d'onde. D'autre part, nous avons défini les cas où l'ouverture est très grande en disant que l'on pouvait les étudier en utilisant les graphiques de transmission avec réfraction et réflexion. Ceci signifie que les phénomènes de diffraction peuvent être considérés comme accessoires. On sait que ces phénomènes tendent à devenir prépondérants lorsque l'ouverture est inférieure à une ou quelques longueurs d'onde. En résumé, on peut dire que les ouvertures moyennes seront celles qui seront comprises entre une fraction sensible de la longueur d'onde et un petit multiple de celle-ci. Disons, pour fixer les idées, un quart de la longueur d'onde et quatre longueurs d'onde. Il est à noter, à propos de cette discussion, que l'ordre de grandeur de l'ouverture est défini à partir de la longueur d'onde et non à partir des dimensions du plan d'eau intérieur.

Il est difficile d'énoncer une règle générale concernant l'influence de l'incidence des ondes excitatrices sur l'agitation à l'intérieur d'un plan d'eau ayant une ouverture d'ordre de grandeur moyen. Il s'agira de cas d'espèce et le plus souvent les études sur plan permettront de dégrossir la question. En tout état de cause, il sera préférable de prévoir, pour les installations d'essais sur modèle, la possibilité de réaliser plusieurs incidences, à moins que l'on se trouve en présence d'un cas exceptionnel où des études théoriques auront permis de limiter les recherches à un angle d'incidence bien défini.

\section{4) Conclusion :}

En résumé, on devra très souvent tenir compte de l'incidence des houles excitatrices même lorsqu'il s'agira d'étudier un port dont l'ouverture sera relativement très étroite. En effet, même si pour un tel port l'incidence n'a pas d'influence directe, elle pourra en avoir sur le mode d'agitation existant à l'extérieur de ce dernier et par conséquent sur l'amplitude existant à son entrée. Nous avons vu, dans le chapitre sur la similitude des modèles réduits destinés à l'étude des seiches portuaires [4], [5], que la nécessité de réaliser des ondes d'incidences différentes est tempérée par le fait que les phénomènes de réfraction rendent l'incidence de celles-ci presque constante au voisinage des côtes pour peu que la topographie des fonds soit assez régulière. Par ailleurs, nous verrons également que la remarque précédente sur la manière dont les ports à ouverture étroite réagissent aux excitalions sans trop tenir compte de leur incidence initiale, peut permettre de notables économies dans certains cas d'étude de seiches en permettant de décomposer l'étude en deux temps:

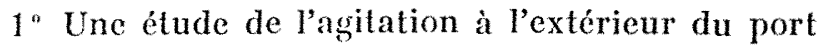
donnant les valeurs de celle-ci à l'entrée;

2" Une étude de l'agitation à l'intérieur du port, 
menée en reconstituant dans un port à plus grande échelle les valeurs de l'agitation trouvées précédemment. Dans certains cas, cette façon de procéder sur modèle peut être si économique qu'elle justifie son application à des cas où l'ouverture représente une fraction assez sensible de la longueur d'onde (tout de même inférieure à la moitié) en considérant une valeur moyenne de l'agitation le long de l'entrée. Là encore, comme c'est toujours le cas pour les études sur modèle, il faut faire un juste départ entre l'intérêt économique d'avoir une solution plus précise et celui de mener une étude moins cồteuse.

BIBLIOGRAPHIE

[1j Biesel-Le Méhauté. - Etude théorique de la houle sur certains obstacles. La Honille Blanche, marsavril 1955 .

[2] BIESEL-Le Méhauté. - Mouvement de résonance à deux dimensions dans une enceinte sous l'action d'ondes incidentes. La Honille Blanche, juillet 1956.

[3] Le Méhauté. - Two dimensional seiche in a basiu subjected to incident waves. Coastal Engineering, 1954 , page 119 .

[4] Biesel-Le Méhauté. - Aperçus sur la similitude des modèles réduits destinés à l'étude des seiches portuaires. La Houille Blanche, juillet 1955.

[5] Bresel. - The similitude of scale models for the study of seiches in harbours. Coastal Engineering, 1954 , page 68
[6] MaNown. - Sur l'entretien des oscillations des eaux portuaires sous l'action de la haute mer. Publications scientifiques et techniques du Ministère de l'Air, 1953.

[7] Biesel-Le Méhauté. - Généralités sur les seiches et les ondes de seiches. La Houille Blanche, octobre-novembre 1955 .

[8] LaM13. - Hydrodynamies.

[9] Bovasse. - Houle, rides, seiches et marées.

10] Kravtchenko-Santon. - Quelques recherches récentes sur les oscillations des eaux portuaires. $6^{\circ}$ Congrès AIRH, La Haye 1955. Communication D 13,1955 .

11] AptÉ. - Recherches théoriques et expérimentales des mouvements des liquides pesants avec surface libre. Thèse, Université de Grenoble, 1955.

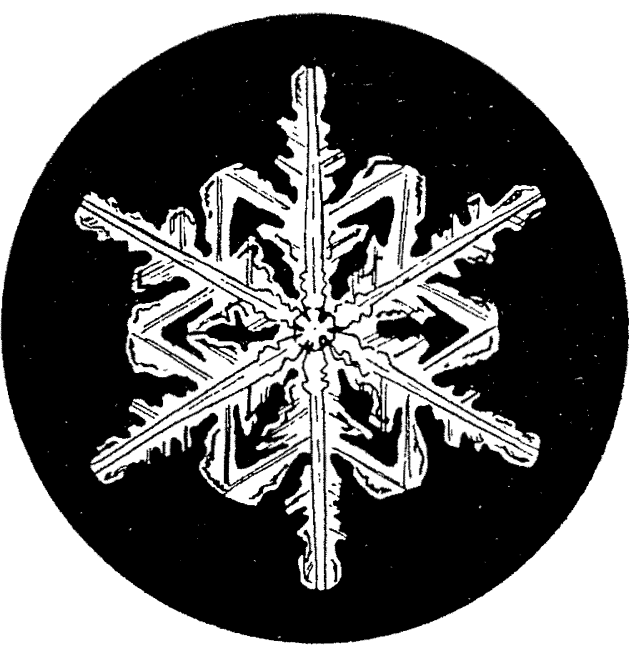

